\title{
Asymptotic behavior of oscillatory solutions of first order functional delay difference equations
}

\author{
A. Murugesan *, C. Soundara Rajan \\ Department of Mathematics, Government Arts College (Autonomous), Salem-636 007, Tamil Nadu, India \\ ${ }^{*}$ Corresponding author E-mail:amurugesan3@gmail.com
}

Copyright (C2015 A. Murugesan and C. Soundara Rajan. This is an open access article distributed under the Creative Commons Attribution License, which permits unrestricted use, distribution, and reproduction in any medium, provided the original work is properly cited.

\section{Abstract}

In this paper, we study the asymptotic behavior of oscillatory solutions of the first order functional delay difference equation

$$
\Delta x(n)=f(n, x(n-\tau)), \quad n \geq n_{0} .
$$

A new sufficient condition is established under which every oscillatory solution of $\left(^{*}\right)$ tends to zero asymptotically.

Keywords: Asymptotic behavior, delay difference equation, oscillatory solution.

\section{Introduction}

In this paper, we consider the following first order functional delay difference equation of the form

$\Delta x(n)=f(n, x(n-\tau)), \quad n \in N\left(n_{0}\right)$

where $\Delta$ is the forward difference operator given by $\Delta x(n)=x(n+1)-x(n), \tau$ is a positive integer, $n_{0}$ is a fixed integer, $N\left(n_{0}\right)=\left\{n_{0}, n_{0}+1, n_{0}+2, \ldots\right\}, f: N\left(n_{0}\right) \times R \rightarrow R$ is a real valued function and for any $n \in N\left(n_{0}\right)$, $f(n,$.$) is a continuous function with the following properties:$

$\left(\mathrm{H}_{1}\right) f(n, 0)=0$;

$\left(\mathrm{H}_{2}\right) u f(n, u)>0$ for $u \neq 0$; and

$\left(\mathrm{H}_{3}\right)$ there exists a sequence $\{q(n)\}$ of positive real numbers defined on $N\left(n_{0}\right)$ such that

$$
|f(n, u)| \leq q(n)|u|
$$

Qualitative theory of discrete processes has drawn considerable attention in recent years. In particular, oscillation properties of discrete analogs of delay differential equations have been studied recently by a number of authors (see e.g., $[6,7,10,11])$. On the other hand, relatively little is known about the asymptotic behavior of all solutions of these discrete equations, see for example [3,8,12], and the references cited therein. For the general background 
of difference equations, one can refer to $[1,2,5,9]$.

In [3], Chen et al. obtained sufficient conditions which ensure that all solutions of the first order nonlinear delay difference equation

$\Delta x(n)+F(n, x(n-k))=0, \quad n \geq n_{0}$

tend to zero as $n \rightarrow \infty$.

In [8], Liu et al. established sufficient conditions under which every solutions of the equation

$\Delta x(n)=p(n) f(x(n-k))+r(n), \quad n=0,1,2, \ldots$

converges to zero. The asymptotic behavior of the solutions of the equation

$\Delta x(n)+p(n) x(n-\tau)=0, \quad n=0,1,2, \ldots$

has been extensively investigated in the literature, see for example, $[4,12,13]$.

The purpose of this paper is to give a new sufficient condition under which every oscillatory solution of (1) tends to zero as $n \rightarrow \infty$. By a solution of (1), we mean a nontrivial real sequence $\{x(n)\}$ which is defined on $N\left(n_{0}-\tau\right)=\left\{n_{0}-\tau, n_{0}-\tau+1, \ldots\right\}$ and which satisfies (1) for $n \in N\left(n_{0}\right)$. A solution $\{x(n)\}$ of $(1)$ on $N\left(n_{0}\right)$ is said to be oscillatory if for every positive integer $N_{0}>n_{0}$, there exists $n \geq N_{0}$ such that $x(n) x(n+1) \leq 0$, otherwise $\{x(n)\}$ is said to be nonoscillatory.

Throughout this paper we use the following notations:

For any $a, b \in N$, define

$N(a)=\{a, a+1, a+2, \ldots\}$,

$N(a, b)=\{a, a+1, a+2, \ldots, b\}$,

$Q(N):=\sup _{n \geq N} \sum_{s=n-\tau}^{n} q(s), \quad$ for $\quad n \geq n_{0}+\tau$

and

$Q_{\infty}:=\lim _{N \rightarrow \infty} Q(N)=\limsup _{n \rightarrow \infty} \sum_{n-\tau}^{n} q(s)$.

\section{Main Results}

Lemma 2.1 Let $\{x(n)\}$ be a solution of (1) and $n_{0}+\tau<n_{1}<n_{2}-1$. If $x(n)>0$ for all $n \in N\left(n_{1}+1, n_{2}-1\right)$ or $x(n)<0$ for all $n \in N\left(n_{1}+1, n_{2}-1\right)$ and $x\left(n_{2}\right) x(n) \leq 0$ for all $n \in N\left(n_{1}+1, n_{2}-1\right)$, then $n_{1} \geq n_{2}-\tau$.

Proof. Assume the contrary, that is, $n_{1}<n_{2}-\tau$. Without loss of generality, we may suppose that $x(n)>0$ for $n \in N\left(n_{1}+1, n_{2}-1\right)$. Then $x\left(n_{2}\right) \leq 0$ and there exists an integer $n^{*}$ satisfying $n_{1} \leq n^{*}-\tau<n^{*}<n_{2}$. Then

$\Delta x(n)=f(n, x(n-\tau))>0$

for $n \in N\left(n^{*}, n_{2}\right)$, which implies $x\left(n_{2}\right)>x\left(n^{*}\right)>0$. This is a contradiction.

The proof is complete.

Lemma 2.2 Given $\delta>0$, there exists an increasing sequence $\{h(n)\}$ of nonnegative real numbers such that

$h(n)-h(n-\tau)=\frac{\delta}{2}, \quad n \geq n_{0}+\tau$. 
Proof. Choose a sequence $\left\{N_{k}\right\}$ of integers such that $N_{0}=n_{0}$ and for $k=0,1,2, \ldots, N_{k+1}=N_{k}+\tau$. Then $\lim _{k \rightarrow \infty} N_{k}=\infty$. Let us define

$h(n)=\frac{\delta}{2}\left(\frac{n-N_{k}}{\tau}+k\right), \quad$ for $\quad n \in N\left(N_{k}, N_{k+1}-1\right)$

for $k=0,1,2, \ldots$. We see that $h\left(N_{k}\right)=\frac{k \delta}{2}$ for all $k$ and $\{h(n)\}$ is an increasing sequence on $N\left(n_{0}\right)$. For any $n \in N\left(N_{k}, N_{k+1}-1\right), k=1,2,3, \ldots$

$h(n)<h\left(N_{k+1}\right) \quad$ and $\quad h(n-\tau) \geq h\left(N_{k}-\tau\right)$,

which implies

$h(n)-h(n-\tau)=\frac{\delta}{2}\left(\frac{n-N_{k}}{\tau}+k\right)-\frac{\delta}{2}\left(\frac{n-\tau-N_{k-1}}{\tau}+k-1\right)=\frac{\delta}{2}$.

Therefore (5) holds for $n \geq N_{1}$.

Theorem 2.3 Let $\{h(n)\}$ be an increasing sequence of positive real numbers satisfying (5) for some $\delta>0$. If $Q(N) \leq \frac{\sqrt{11}-1}{2}$ for some $N \geq n_{0}+\tau$, then for any oscillatory solution $\{x(n)\}$ of $(1)$, there exists a $K=K(\beta, h, x)>$ 0 such that

$|x(n)|<K \bar{e}^{\beta h(n)}, \quad n \in N\left(n_{0}\right)$,

where

$$
\left.\begin{array}{rl}
\beta=\frac{2}{3 \delta} \log \frac{4}{(Q(N)+1)^{2}-1}, & Q(N)<1 \\
\beta=\frac{2}{3 \delta} \log \frac{2}{\left(Q(N)+\frac{1}{2}\right)^{2}-\frac{3}{4}}, & 1 \leq Q(N) \leq \frac{\sqrt{11}-1}{2} .
\end{array}\right\}
$$

Proof. Since $\{x(n)\}$ is an oscillatory solution of (1), there exists a sufficiently large $n^{*}>N_{0}+\tau$ such that $x\left(n^{*}\right) \leq 0$. We will show that (6) holds for a positive constant $K$ such that

$K>\max _{n_{0} \leq n \leq n^{*}} e^{\beta h(n)}|x(n)|$.

Assume that (6) does not hold. Then there exists an integer $\xi>n^{*}$ such that

$|x(n)|<K \bar{e}^{\beta h(n)} \quad$ for $n \in N\left(n_{0}, \xi-1\right) \quad$ and $\quad|x(\xi)| \geq K \bar{e}^{\beta h(\xi)}$.

Then $x(\xi) \neq 0$. Since $\{x(n)\}$ is oscillatory and $\xi>n^{*}$, we can define two integers $n_{1}, n_{2} \in N\left(n_{0}\right)$ by

$n_{1}=\sup \{n: n<\xi, \quad x(n) x(\xi) \leq 0\}$

and

$n_{2}=\inf \{n: n>\xi, \quad x(n) x(\xi) \leq 0\}$.

We see that $n^{*} \leq n_{1}<\xi<n_{2}$ and $x(n)>0$ for all $n \in N\left(n_{1}+1, n_{2}-1\right)$ or $x(n)<0$ for all $n \in N\left(n_{1}+1, n_{2}-1\right)$. Also, we have $x\left(n_{1}\right) x(n) \leq 0$ for all $n \in N\left(n_{1}+1, n_{2}-1\right)$ and $x\left(n_{2}\right) x(n) \leq 0$ for all $n \in N\left(n_{1}+1, n_{2}-1\right)$. Lemma 2.1 leads to $n_{1} \geq n_{2}-\tau$ and hence $n_{1} \geq u-\tau$ for $u \in N\left(n_{1}, n_{2}\right)$. Then

$$
\begin{aligned}
|x(u-\tau)| & =\left|x(u-\tau)-x\left(n_{1}\right)+x\left(n_{1}\right)\right| \\
& \leq\left|x\left(n_{1}\right)-x(u-\tau)\right|+\left|x\left(n_{1}\right)\right| \\
& =\left|\sum_{s=u-\tau}^{n_{1}-1} \Delta x(s)\right|+\left|x\left(n_{1}\right)\right| \\
& =\left|\sum_{s=u-\tau}^{n_{1}-1} f(s, x(s-\tau))\right|+\left|x\left(n_{1}\right)\right|
\end{aligned}
$$


which implies that for $u \in N\left(n_{1}, n_{2}\right)$,

$|x(u-\tau)| \leq \sum_{s=u-\tau}^{n_{1}-1} q(s)|x(s-\tau)|+\left|x\left(n_{1}\right)\right|$.

Moreover, because of $x\left(n_{1}\right) x(\xi) \leq 0$, we have

$$
\begin{aligned}
|x(\xi)| & \leq\left|x(\xi)-x\left(n_{1}\right)\right| \\
& =\left|\sum_{u=n_{1}}^{\xi-1} \Delta x(u)\right| \\
& =\left|\sum_{u=n_{1}}^{\xi-1} f(u, x(u-\tau))\right| \\
& \leq \sum_{u=n_{1}}^{\xi-1} q(u)|x(u-\tau)|,
\end{aligned}
$$

and

$$
\begin{aligned}
|x(\xi)| & \leq\left|x\left(n_{2}\right)-x(\xi)\right| \\
& =\left|\sum_{u=\xi}^{n_{2}-1} \Delta x(u)\right| \\
& \leq\left|\sum_{u=\xi}^{n_{2}-1} f(u, x(u-\tau))\right| \\
& \leq \sum_{u=\xi}^{n_{2}-1} q(u)|x(u-\tau)| .
\end{aligned}
$$

Thus we obtain

$$
|x(\xi)| \leq \frac{1}{2} \sum_{u=n_{1}}^{n_{2}-1} q(u)|x(u-\tau)| .
$$

Here we consider two cases.

Case 1: $Q(N)<1$. We note that $\beta>0$. By (9) and (10),

$$
|x(\xi)| \leq \frac{1}{2} \sum_{u=n_{1}}^{n_{2}-1} q(u)\left(\sum_{s=u-\tau}^{n_{1}-1} q(s)|x(s-\tau)|+\left|x\left(n_{1}\right)\right|\right)
$$

or

$$
|x(\xi)| \leq \frac{1}{2} \sum_{u=n_{1}}^{n_{2}-1} q(u) \sum_{s=u-\tau}^{n_{1}-1} q(s)|x(s-\tau)|+\frac{1}{2}\left|x\left(n_{1}\right)\right| \sum_{u=n_{1}}^{n_{2}-1} q(u)
$$

By (8), we see that $|x(s-\tau)|<K \bar{e}^{\beta h(s-\tau)}$ for $s \in N\left(n_{0}+\tau, n_{1}\right)$. Then by (11),

$$
|x(\xi)|<\frac{1}{2} \sum_{u=n_{1}}^{n_{2}-1} q(u) \sum_{s=u-\tau}^{n_{1}-1} q(s) K \bar{e}^{\beta h(s-\tau)}+\frac{1}{2}\left|x\left(n_{1}\right)\right| \sum_{u=n_{1}}^{n_{2}-1} q(u) .
$$

Since $\{h(n)\}$ is increasing and $\beta>0$,

$|x(\xi)|<\frac{K}{2} \sum_{u=n_{1}}^{n_{2}-1} q(u) \bar{e}^{\beta h(u-2 \tau)} \sum_{s=u-\tau}^{n_{1}-1} q(s)+\frac{1}{2}\left|x\left(n_{1}\right)\right| \sum_{u=n_{1}}^{n_{2}-1} q(u)$ 
$\leq \frac{K}{2} \bar{e}^{\beta h\left(n_{1}-2 \tau\right)} \sum_{u=n_{1}}^{n_{2}-1} q(u) \sum_{s=u-\tau}^{n_{1}-1} q(s)+\frac{1}{2}\left|x\left(n_{1}\right)\right| \sum_{u=n_{1}}^{n_{2}-1} q(u)$.

Then

$|x(\xi)|<\frac{K}{2} \bar{e}^{\beta h\left(n_{1}-2 \tau\right)}\left\{\sum_{u=n_{1}}^{n_{2}-1} q(u) \sum_{s=u-\tau}^{u} q(s)-\sum_{u=n_{1}}^{n_{2}-1} q(u) \sum_{s=n_{1}}^{u} q(s)\right\}+\left|x\left(n_{1}\right)\right| \sum_{u=n_{1}}^{n_{2}-1} q(u)$.

Since

$\sum_{u=n_{1}}^{n_{2}-1} q(u) \sum_{s=n_{1}}^{u} q(s) \geq \frac{1}{2} \sum_{u=n_{1}}^{n_{2}-1} q(u) \sum_{s=n_{1}}^{n_{2}-1} q(s)=\frac{1}{2}\left(\sum_{u=n_{1}}^{n_{2}-1} q(u)\right)^{2}$

$|x(\xi)|<\frac{K}{2} \bar{e}^{\beta h\left(n_{1}-2 \tau\right)}\left\{Q(N) \sum_{u=n_{1}}^{n_{2}-1} q(u)-\frac{1}{2}\left(\sum_{u=n_{1}}^{n_{2}-1} q(u)\right)^{2}\right\}+\frac{1}{2}\left|x\left(n_{1}\right)\right| Q(N)$.

The right side of (13) is a quadratic function of

$\sum_{u=n_{1}}^{n_{2}-1} q(u) \quad$ and $\quad 0<\sum_{u=n_{1}}^{n_{2}-1} q(u) \leq \sum_{u=n_{2}-\tau}^{n_{2}-1} q(u) \leq Q(N)$.

Then

$$
\begin{aligned}
|x(\xi)| & <\frac{K}{2} \bar{e}^{\beta h\left(n_{1}-2 \tau\right)}\left\{Q^{2}(N)-\frac{Q^{2}(N)}{2}\right\}+\frac{K}{2} \bar{e}^{\beta h\left(n_{1}-2 \tau\right)} Q(N) \\
& =\frac{K}{2} \bar{e}^{\beta h\left(n_{1}-2 \tau\right)}\left\{\frac{Q^{2}(N)}{2}+Q(N)\right\} \\
& =\frac{K}{4} \bar{e}^{\beta h\left(n_{1}-2 \tau\right)}\left\{Q^{2}(N)+2 Q(N)\right\} \\
& \leq \frac{K}{4} \bar{e}^{\beta h(\xi-3 \tau)}\left\{(Q(N)+1)^{2}-1\right\} \\
& \leq \frac{K}{4}\left\{(Q(N)+1)^{2}-1\right\} \bar{e}^{\beta h(\xi-3 \tau)} \\
& =\frac{K}{4}\left\{(Q(N)+1)^{2}-1\right\} e^{\beta(h(\xi)-h(\xi-3 \tau))} e^{-\beta h(\xi)} .
\end{aligned}
$$

By Lemma 2.2, $h(\xi)-h(\xi-3 \tau)=\frac{3 \delta}{2}$.

Then, we have

$|x(\xi)|<\frac{1}{4}\left\{(Q(N)+1)^{2}-1\right\} e^{\frac{3 \delta \beta}{2}}\left(K e^{-\beta h(\xi)}\right)$.

Thus, (7) implies $|x(\xi)|<K \bar{e}^{\beta h(\xi)}$.

Then we have a contradiction to the assumption that $|x(\xi)| \geq K \bar{e}^{\beta h(\xi)}$.

Case 2: $1 \leq Q(N) \leq \frac{\sqrt{11}-1}{2}$. We note that $\beta \geq 0$. There are two possibilities.

Case 2.1: $1 \leq \sum_{n=n_{1}}^{n_{2}-1} q(n) \leq \frac{\sqrt{11}-1}{2}$. There exists an integer $\eta$ such that $n_{1} \leq \eta \leq n_{2}-1$ and $\sum_{n=\eta}^{n_{2}-1} q(n) \geq 1$. By (9) and (10), we have

$|x(\xi)| \leq \frac{1}{2} \sum_{u=n_{1}}^{n_{2}-1} q(u)|x(u-\tau)|$ 


$$
\begin{aligned}
& \leq \frac{1}{2} \sum_{u=n_{1}}^{\eta-1} q(u)|x(u-\tau)|+\frac{1}{2} \sum_{u=\eta}^{n_{2}-1} q(u)|x(u-\tau)| \\
& \leq \frac{1}{2} \sum_{u=n_{1}}^{\eta-1} q(u)|x(u-\tau)|+\frac{1}{2} \sum_{u=\eta}^{n_{2}-1} q(u)\left\{\sum_{s=u-\tau}^{n_{1}-1} q(s)|x(s-\tau)|+\left|x\left(n_{1}\right)\right|\right\} \\
= & \frac{1}{2} \sum_{u=n_{1}}^{\eta-1} q(u)|x(u-\tau)|+\frac{1}{2} \sum_{u=\eta}^{n_{2}-1} q(u) \sum_{s=u-\tau}^{n_{1}-1} q(s)|x(s-\tau)|+\frac{1}{2} \sum_{u=\eta}^{n_{2}-1} q(u)\left|x\left(n_{1}\right)\right| \\
= & \frac{1}{2} \sum_{u=n_{1}}^{\eta-1} q(u)|x(u-\tau)|+\frac{1}{2} \sum_{u=\eta}^{n_{2}-1} q(u)\left[\sum_{s=u-\tau}^{\eta-1} q(s)|x(s-\tau)|-\sum_{s=n_{1}}^{\eta-1} q(s)|x(s-\tau)|\right]+\frac{1}{2} \sum_{u=\eta}^{n_{2}-1} q(u)\left|x\left(n_{1}\right)\right| \\
= & \frac{1}{2} \sum_{u=n_{1}}^{\eta-1} q(u)|x(u-\tau)|+\frac{1}{2} \sum_{u=\eta}^{n_{2}-1} q(u) \sum_{s=u-\tau}^{\eta-1} q(s)|x(s-\tau)|-\frac{1}{2} \sum_{u=\eta}^{n_{2}-1} q(u) \sum_{s=n_{1}}^{\eta-1} q(s)|x(s-\tau)|+\frac{1}{2} \sum_{u=\eta}^{n_{2}-1} q(u)\left|x\left(n_{1}\right)\right| .
\end{aligned}
$$

Since $\sum_{n=\eta}^{n_{2}-1} q(n) \geq 1$,

$$
\sum_{u=\eta}^{n_{2}-1} q(u) \sum_{s=n_{1}}^{\eta-1} q(s)|x(s-\tau)|=\left(\sum_{u=\eta}^{n_{2}-1} q(u)\right)\left(\sum_{s=n_{1}}^{\eta-1} q(s)|x(s-\tau)|\right) \geq \sum_{s=n_{1}}^{\eta-1} q(s)|x(s-\tau)|
$$

which implies

$$
|x(\xi)| \leq \frac{1}{2} \sum_{u=\eta}^{n_{2}-1} q(u) \sum_{s=u-\tau}^{\eta-1} q(s)|x(s-\tau)|+\frac{1}{2} \sum_{u=\eta}^{n_{2}-1} q(u)\left|x\left(n_{1}\right)\right| .
$$

By (8) and the fact that $s-\tau \leq n_{1}<\xi$ for $s \in N\left(n_{0}+\tau, n_{2}\right),|x(s-\tau)|<K e^{-\beta h(s-\tau)}$ for $s \in N\left(n_{0}+\tau, \eta\right)$. Then by (14),

$$
\begin{aligned}
|x(\xi)| & <\frac{1}{2} \sum_{u=\eta}^{n_{2}-1} q(u) \sum_{s=u-\tau}^{\eta-1} q(s) K \bar{e}^{\beta h(s-\tau)}+\frac{K}{2} e^{-\beta h\left(n_{1}-\tau\right)} \sum_{u=\eta}^{n_{2}-1} q(u) \\
& \leq \frac{K}{2} \sum_{u=\eta}^{n_{2}-1} q(u) \bar{e}^{\beta(u-2 \tau)} \sum_{s=u-\tau}^{\eta-1} q(s)+\frac{K}{2} e^{-\beta h\left(n_{1}-\tau\right)} \sum_{s=u-\tau}^{\eta-1} q(s) \\
& \leq \frac{K}{2} \bar{e}^{\beta h\left(n_{1}-2 \tau\right)}\left\{\sum_{u=\eta}^{n_{2}-1} q(u) \sum_{s=u-\tau}^{\eta-1} q(s)+\sum_{s=u-\tau}^{\eta-1} q(s)\right\} \\
& \leq \frac{K}{2} \bar{e}^{\beta h\left(n_{1}-2 \tau\right)}\left\{\sum_{u=\eta}^{n_{2}-1} q(u) \sum_{s=u-\tau}^{\eta-1} q(s)+Q(N)\right\} \\
& =\frac{K}{2} \bar{e}^{\beta h\left(n_{1}-2 \tau\right)}\left\{\sum_{u=\eta}^{n_{2}-1} q(u) \sum_{s=u-\tau}^{u} q(s)-\sum_{u=\eta}^{n_{2}-1} q(u) \sum_{s=\eta}^{u} q(s)+Q(N)\right\} .
\end{aligned}
$$

Since

$$
\begin{aligned}
\sum_{u=\eta}^{n_{2}-1} q(u) \sum_{s=\eta}^{u} q(s) & \geq \frac{1}{2}\left(\sum_{u=\eta}^{n_{2}-1} q(u)\right)^{2}, \\
|x(\xi)| & <\frac{K}{2} \bar{e}^{\beta h\left(n_{1}-2 \tau\right)}\left\{Q(N) \sum_{u=\eta}^{n_{2}-1} q(u)-\frac{1}{2}\left(\sum_{u=\eta}^{n_{2}-1} q(u)\right)^{2}+Q(N)\right\} \\
& \leq \frac{K}{2} \bar{e}^{\beta h\left(n_{1}-2 \tau\right)}\left\{Q^{2}(N)-\frac{1}{2}+Q(N)\right\}
\end{aligned}
$$




$$
\begin{aligned}
& \leq \frac{K}{2} \bar{e}^{\beta h\left(n_{1}-2 \tau\right)}\left\{\left(Q(N)+\frac{1}{2}\right)^{2}-\frac{3}{4}\right\} \\
& \leq \frac{K}{2} \bar{e}^{\beta h(\xi-3 \tau)}\left\{\left(Q(N)+\frac{1}{2}\right)^{2}-\frac{3}{4}\right\} \\
& =\frac{K}{2} e^{\beta(h(\xi)-h(\xi-3 \tau))} e^{-\beta h(\xi)}\left\{\left(Q(N)+\frac{1}{2}\right)^{2}-\frac{3}{4}\right\} \\
& =\frac{K}{2} e^{\frac{3 \delta \beta}{2}} e^{-\beta h(\xi)}\left\{\left(Q(N)+\frac{1}{2}\right)^{2}-\frac{3}{4}\right\} .
\end{aligned}
$$

Thus, (7) implies $|x(\xi)|<K \bar{e}^{\beta h(\xi)}$. Then we have a contradiction to the assumption that $|x(\xi)| \geq K \bar{e}^{\beta h(\xi)}$.

Case 2.2: $\sum_{n=n_{1}}^{n_{2}-1} q(n)<1$. In the same way as a Case 1 , we have

$|x(\xi)|<\frac{K}{2} \bar{e}^{\beta h\left(n_{1}-2 \tau\right)}\left\{Q(N) \sum_{u=n_{1}}^{n_{2}-1} q(u)-\frac{1}{2}\left(\sum_{u=n_{1}}^{n_{2}-1} q(u)\right)^{2}+Q(N)\right\}$.

Since $Q(N) \sum_{u=n_{1}}^{n_{2}-1} q(u)-\frac{1}{2}\left(\sum_{u=n_{1}}^{n_{2}-1} q(u)\right)^{2}$ is a quadratic function of $\sum_{u=n_{1}}^{n_{2}-1} q(u)$ and $0<\sum_{u=n_{1}}^{n_{2}-1} q(u)<1 \leq Q(N)$, we have,

$$
\begin{aligned}
|x(\xi)| & <\frac{K}{2} \bar{e}^{\beta h\left(n_{1}-2 \tau\right)}\left\{Q(N) \cdot 1-\frac{1}{2} \cdot 1^{2}+Q(N)\right\} \\
& \leq \frac{K}{2} \bar{e}^{\beta h\left(n_{1}-2 \tau\right)}\left\{Q^{2}(N)+Q(N)-\frac{1}{2}\right\} \\
& \leq \frac{K}{2} \bar{e}^{\beta h(\xi-3 \tau)}\left\{\left(Q(N)+\frac{1}{2}\right)^{2}-\frac{3}{4}\right\} \\
& =\frac{K}{2} e^{\beta(h(\xi)-h(\xi-3 \tau))} e^{-\beta h(\xi)}\left\{\left(Q(N)+\frac{1}{2}\right)^{2}-\frac{3}{4}\right\} \\
& =\frac{K}{2} e^{\frac{3 \delta \beta}{2}} e^{-\beta h(\xi)}\left\{\left(Q(N)+\frac{1}{2}\right)^{2}-\frac{3}{4}\right\} .
\end{aligned}
$$

By $(7),|x(\xi)|<K \bar{e}^{\beta h(\xi)}$. Then we have a contradiction to the assumption that $|x(\xi)| \geq K \bar{e}^{\beta h(\xi)}$. Hence, by virtue of the Case 1 and 2, we obtain (6). The proof is complete.

Using Theorem 2.3, we have two corollaries.

Corollary 2.4 If $Q(N) \leq \frac{\sqrt{11}-1}{2}$ for some $N \geq n_{0}+\tau$, then every oscillatory solution of (1) is bounded.

Corollary 2.5 If $Q_{\infty}<\frac{\sqrt{11}-1}{2}$, then every oscillatory solution of (1) tends to zero as $n \rightarrow \infty$.

In the case where $\frac{\sqrt{11}-1}{2}<Q(N)<\infty$ for some $N \geq n_{0}+\tau$ we can prove the following theorem in the same way as case 1 in the proof of Theorem 2.3:

Theorem 2.6 If $\{h(n)\}$ is an increasing sequence of positive real numbers defined on $N\left(n_{0}\right)$ and $Q(N)>\frac{\sqrt{11}-1}{2}$ for some $N \geq n_{0}+\tau$, then for any oscillatory solution $\{x(n)\}$ of (1), there exists a constant $K=K(h, x)>0$ such that

$|x(n)|<K e^{h(n)}, \quad n \geq n_{0}$. 
Lemma 2.7 Let $f(n, x(n-\tau)=q(n) x(n-\tau)$, where $\{q(n)\}$ is a sequence of nonnegative and real numbers defined on $N\left(n_{0}\right),\{x(n)\}$ be a solution of (1) and $Q_{\infty}<\infty$. If $\{x(n)\}$ is not oscillatory, then there exist a $N_{0}=N_{0}(x)>n_{0}$ and a positive constant $C=C(x)$ such that

$|x(n)| \geq C \exp \left\{\frac{1}{Q_{\infty}+2} \sum_{s=n_{0}}^{n-1} q(s)\right\}, \quad n>N_{0}$.

Proof. Without loss of generality, we may assume that $\{x(n)\}$ is eventually positive, i.e., there exists $n_{1}>n_{0}$ such that $x(n)>0$ for any $n>n_{1}$. Choose $N_{0}>n_{0}$, such that $N_{0}-3 \tau>n_{1}$ and $\sum_{s=n-\tau}^{n} q(s)<Q_{\infty}+1, \quad n>N_{0}$.

By $(1)$ and $x(n) \neq 0$ for $n>n_{1}$, we have

$x(n) \geq x\left(N_{0}\right) \exp \left\{\sum_{s=N_{0}}^{n-1} q(s) \frac{x(s-\tau)}{x(s+1)}\right\}, \quad n>N_{0}$.

Since $\Delta x(n) \geq 0$ for $n>n_{1}+\tau$, we have

$$
\begin{aligned}
x(n+1)-x(n-\tau) & =\sum_{s=n-\tau}^{n} \Delta x(s) \\
& =\sum_{s=n-\tau}^{n} q(s) x(s-\tau) \\
& \leq x(n-\tau) \sum_{s=n-\tau}^{n} q(s) \\
& \leq x(n-\tau)\left(Q_{\infty}+1\right), \quad n>N_{0} .
\end{aligned}
$$

Then

$\frac{x(n-\tau)}{x(n+1)} \geq \frac{1}{Q_{\infty}+2}$,

which leads to that

$$
\begin{aligned}
x(n) & \geq x\left(N_{0}\right) \exp \left\{\frac{1}{Q_{\infty}+2} \sum_{s=N_{0}}^{n-1} q(s)\right\} \\
& =x\left(N_{0}\right) \exp \left\{\frac{-1}{Q_{\infty}+2} \sum_{s=n_{0}}^{N_{0}-1} q(s)\right\} \exp \left\{\frac{1}{Q_{\infty}+2} \sum_{s=n_{0}}^{n-1} q(s)\right\}, \quad n>N_{0} .
\end{aligned}
$$

The proof is complete. By this lemma we obtain the following theorem:

Theorem 2.8 Assume that $Q_{\infty}<\frac{\sqrt{11}-1}{2}$.

(i) If a solution $\{x(n)\}$ of (1) satisfies

$$
\lim _{n \rightarrow \infty} \frac{x(n)}{\exp \left\{\frac{1}{Q_{\infty}+2} \sum_{s=n_{0}}^{n-1} q(s)\right\}}=0,
$$

then $x(n)$ tends to zero as $n \rightarrow \infty$.

(ii) If

$$
\sum_{s=n_{0}}^{\infty} q(s)=\infty
$$

then every bounded solution of (1) tends to zero as $n \rightarrow \infty$.

Proof. (i) By Lemma 2.7 and (15), $\{x(n)\}$ is oscillatory. Therefore, by Corollary 2.5, $x(n)$ tends to zero as $n \rightarrow \infty$.

(ii) Let $\{x(n)\}$ be a bounded solution of (1). By (16), $\{x(n)\}$ satisfies (15).

Hence $x(n)$ tends to zero as $n \rightarrow \infty$.

The proof is complete. 


\section{Equations with special forcing term}

Let $A_{0}$ be the set of all real sequences $\{a(n)\}$ defined on $N\left(n_{0}-\tau\right)$ such that

$\lim _{n \rightarrow \infty} a(n)=0$.

Consider the following equation:

$\Delta x(n)=q(n) x(n-\tau)+r(n), \quad n \geq n_{0}$,

where the sequence $\{r(n)\}$ is given by $r(n)=q(n) a(n-\tau)-\Delta a(n)$ with some $\{a(n)\} \in A_{0}$. We compare the asymptotic behavior of the oscillatory solution of (17) with that of the equation (1).

Lemma 3.1 Let $\{y(n)\}$ be a sequence of real numbers defined on $N\left(n_{0}\right)$ and $\{a(n)\} \in A_{0}$. If $z(n)=y(n)+a(n)$ is a solution of (1) and $\{y(n)\}$ is oscillatory, then $\{z(n)\}$ is also oscillatory.

Proof. Assume that $\{z(n)\}$ is not oscillatory. Then there exists $n_{1}>n_{0}+\tau$ such that $|z(n)|>0$ for $n>n_{1}$. Without loss of generality, we may assume that $z(n)>0$. Since $\{z(n)\}$ is a solution of (1) and $q(n) \geq 0, \Delta z(n)=$ $q(n) z(n-\tau) \geq 0$ for $n>n_{2}$ for some $n_{2}>n_{1}+\tau$. Then $z(n) \geq z\left(n_{2}\right)>0$ for $n>n_{2}$. Since $\{a(n)\}$ tends to zero as $n \rightarrow \infty$, we have

$\liminf _{n \rightarrow \infty} y(n)=\liminf _{n \rightarrow \infty}\{z(n)-a(n)\} \geq z\left(n_{2}\right)>0$.

This is a contradiction to the assumption that $\{y(n)\}$ is oscillatory. The proof is complete.

Theorem 3.2 If every oscillatory solution of (1) tends to zero as $n \rightarrow \infty$, then every oscillatory solution of (17) tends to zero as $n \rightarrow \infty$.

Proof. Let $\{x(n)\}$ be an oscillatory solution of (17). Then it follows that

$\Delta x(n)=q(n) x(n-\tau)+q(n) a(n-\tau)-\Delta a(n)$,

which implies that

$\Delta(x(n)+a(n))=q(n)(x(n-\tau)+a(n-\tau))$.

Set

$z(n)=x(n)+a(n)$.

Then $\{z(n)\}$ is a solution of (1), we see from Lemma 3.1 that $\{z(n)\}$ is oscillatory. Therefore $z(n)$ tends to zero as $n \rightarrow \infty$ by assumption. Hence $x(n)=z(n)-a(n)$ tends to zero as $n \rightarrow \infty$.

The proof is complete.

Corollary 3.3 If $Q_{\infty}<\frac{\sqrt{11}-1}{2}$, then every oscillatory solution of (17) tends to zero as $n \rightarrow \infty$.

Proof. By Corollary 2.5, every oscillatory solution of (1) tends to zero as $n \rightarrow \infty$. By Theorem 3.2, we obtain that every oscillatory solution of (17) tends to zero as $n \rightarrow \infty$.

The proof is complete. Now, let us consider the equations:

$\Delta x(n)=q x(n-\tau)+\mu \lambda^{-n}, \quad n \geq n_{0}$

and

$\Delta x(n)=q x(n-\tau)$

where $\mu$ is a constant, $\tau$ is a positive integer and $q, \lambda$ are positive real numbers with $\lambda>1$.

Theorem 3.4 Every oscillatory solution of (18) tends to zero as $n \rightarrow \infty$, if and only if every oscillatory solution of (19) tends to zero as $n \rightarrow \infty$. 
Proof. Sufficiency. Suppose that every oscillatory solution of (19) tends to zero as $n \rightarrow \infty$. Define a sequence $\{a(n)\}$ on $N\left(n_{0}-\tau\right)$ by

$a(n)=\frac{\mu \lambda^{-n}}{1-\frac{1}{\lambda}+q \lambda^{\tau}}$.

Then we see that $\{a(n)\} \in A_{0}$ and $\mu \lambda^{-n}=q a(n-\tau)-\Delta a(n)$. By Theorem 3.2 every oscillatory solution of (18) tends to zero as $n \rightarrow \infty$.

Necessity. Suppose that every oscillatory solution of (18) tends to zero as $n \rightarrow \infty$ for some $\mu \neq 0$. Let $\{y(n)\}$ be an oscillatory solution of (19) and let $z(n)=y(n)-a(n)$. Then

$\Delta z(n)=q z(n-\tau)+q a(n-\tau)-\Delta a(n)$

$=q z(n-\tau)+\mu \lambda^{-n}$,

which means that $\{z(n)\}$ is a solution of (18), We will prove that $z(n)$ tends to zero as $n \rightarrow \infty$. If $\{z(n)\}$ is oscillatory, then $z(n)$ tends to zero as $n \rightarrow \infty$ by assumption. Therefore it is enough to consider the case that $\{z(n)\}$ is not oscillatory. We will show that for some $N^{*}>n_{0}$,

$0<|z(n)| \leq \frac{|\mu|}{1-\frac{1}{\lambda}} \lambda^{-n}, \quad n>N^{*}$.

Let $\mu>0$. Assume that $z(n)>0$ for $n>N_{1}$ for some $N_{1}>n_{0}$, Since $q z(n-\tau)>0$ for $n>N_{1}+\tau, \Delta z(n)>0$ for $n>N_{1}+\tau$. Then $\{z(n)\}$ is monotonic increasing as $N\left(N_{1}+\tau\right)$, which implies that for $N_{2}>N_{1}+\tau$,

$\liminf _{n \rightarrow \infty} y(n) \geq \liminf _{n \rightarrow \infty} z(n)+\liminf _{n \rightarrow \infty} a(n)$

$\geq z\left(N_{2}\right)>0$.

Since $\{y(n)\}$ is oscillatory, we have a contradiction. Hence $z(n)<0$ for $n>N_{3}$ for some $N_{3}>n_{0}$. Then

$\Delta\left(z(n)+\frac{\mu \lambda^{-n}}{1-\frac{1}{\lambda}}\right)=q z(n-\tau)<0, \quad n>N_{3}+\tau$,

which implies that $\left\{z(n)+\frac{\mu \lambda^{-n}}{1-\frac{1}{\lambda}}\right\}$ is monotone decreasing on $N\left(N_{3}+\tau\right)$. Then we have that for any $m>N_{3}+\tau$,

$\limsup _{n \rightarrow \infty} y(n)=\limsup _{n \rightarrow \infty}(z(n)+a(n))$

$\leq\left(\limsup _{n \rightarrow \infty} z(n)+\frac{\mu \lambda^{-n}}{1-\frac{1}{\lambda}}\right)+\limsup _{n \rightarrow \infty}\left(a(n)-\frac{\mu \lambda^{-n}}{1-\frac{1}{\lambda}}\right)$

$\leq z(m)+\frac{\mu \lambda^{-m}}{1-\frac{1}{\lambda}}$

Since $\lim \sup _{n \rightarrow \infty} y(n) \geq 0, z(m)+\frac{\mu \lambda^{-m}}{1-\frac{1}{\lambda}} \geq 0$ for $m>N_{3}+\tau$. Therefore $0>z(n) \geq \frac{-\mu \lambda^{-m}}{1-\frac{1}{\lambda}}, n>N_{3}+\tau$. In case $\mu<0$, we see in the same way that $0<z(n) \leq \frac{-\mu \lambda^{-m}}{1-\frac{1}{\lambda}}, n>N_{4}$, for some $N_{4}>n_{0}$. Then we have (20), and hence $z(n)$ tends to zero as $n \rightarrow \infty$ when $z(n)$ is not oscillatory. Therefore $y(n)=z(n)+a(n)$ tends to zero as $n \rightarrow \infty$, which implies that every oscillatory solution of (19) tends to zero as $n \rightarrow \infty$.

The proof is complete.

\section{References}

[1] R. P. Agarwal, Advanced Topics in Difference Equations, Kluwer Academic Publishers Inc., 1997.

[2] R. P. Agarwal, Difference Equations and Inequalities: Theory, Methods, and Applications, Marcel Dekker, Inc., New York, 1999. 
[3] M. P. Chen and B. Liu, Asymptotic behavior of solutions of first order nonlinear delay difference equations, Comput. Math. Appl., 32(1996), 9-13.

[4] L. H. Erbe, H. Xian and J. S. Yu, Global stability of a linear nonautonomous delay difference equation, J. Difference Equ. Appl., 1(1995), 151-161.

[5] I. Györi and G. Ladas, Oscillation Theory of Delay Differential Equations with Applications, Oxford University Press, Oxford, (1991).

[6] G. Ladas, Explicit conditions for the oscillation of difference equations, Math. Anal. Appl., 153(1990), $276-287$.

[7] B. S. Lalli, Oscillation theorems for neutral difference equations, Comput. Math. Appl., 28(1994), $191-202$.

[8] Y. Liu and W. Ge, Global asymptotic behavior of solutions of a forced delay difference equation, Comput. Math. Appl., 47(2004), 1211-1224.

[9] R. E. Mickens, Difference Equations, Van Nostrand Reinhold Company Inc., New York 1987.

[10] Ch. G. Philos, On oscillations of some difference equations, Funkcialaj Ekvacioj, 34(1991), 157-172.

[11] X. H. Tang and J. S. Yu, A further result on the oscillation of delay difference equations, Comput. Math. Appl., 38(1999), 229-237.

[12] J. S. Yu, Asymptotic stability for a linear difference equation with variable delay, Comput. Math. Appl., 36(1998), 202-210.

[13] Z. Zhou, J. S. Yu and Z. C. Wang, Global attractivity of neutral difference equations, Comput. Math. Appl., 36(6)(1998), $1-10$. 